Comparative Population Studies

Jg. 41 (2016): D1-D10 (Erstveröffentlichung: 22.08.2017)

\title{
Das Modell des Zensus 2011
}

\author{
Sabine Bechtold
}

\section{$1 \quad$ Der Zensus 2011 im Überblick}

Das Grundprinzip der traditionellen Volkszählungen, wie sie vor dem Zensus 2011 in Deutschland stattfanden, ist sehr leicht zu verstehen. Für jede am jeweiligen Stichtag in Deutschland lebende Person wurden mit Hilfe eines Papierfragebogens alle Informationen erhoben, die im Rahmen der jeweiligen Volkszählung zu erfassen waren. Dies fand entweder als persönliche Befragung durch Erhebungsbeauftragte statt, oder durch Selbstausfüllung des Fragebogens, den Erhebungsbeauftragte persönlich abgegeben hatten, wobei für die Volkszählungen jeweils Teilnahmepflicht bestand. Das Ergebnis war ein Einzeldatenbestand, der für jede Person einen Datensatz mit allen Volkszählungsmerkmalen enthielt. Wie jede statistische Erhebungen waren auch diese Volkszählungen nicht fehlerfrei, allerdings gab es nur systematische Fehler und keine Zufallsfehler, welche nur bei Stichprobenerhebungen auftreten.

Beim Zensus 2011 wurden im Gegensatz zu diesem sehr gradlinigen Ansatz viele verschiedene Datenquellen und Methoden herangezogen, um Ergebnisse zu Personen, Haushalten sowie Gebäuden und Wohnungen zu erhalten. Das Modell umfasst sowohl Registerdaten als auch Erhebungsdaten aus schriftlichen und persönlichen Befragungen, die als Voll- oder als Stichprobenerhebungen - jeweils mit Auskunftspflicht - konzipiert waren, sowie Datenzusammenführungen und Verfahren der maschinellen Datengenerierung. Grundidee des Zensus 2011 war, zunächst von den in den Melderegistern enthaltenen demografischen Grunddaten auszugehen und diese dann mit Daten aus anderen Registern oder aus Primärerhebungen zu ergänzen und ggf. zu korrigieren. ${ }^{1}$ Im Ergebnis gibt es für jede mit dem Zensus zu erfassende Person sowie für jedes Gebäude mit Wohnraum und jede Wohnung einen eindeutigen Datensatz mit allen geforderten Zensusmerkmalen. Das Gleiche gilt für jeden Haushalt, der im Zuge des maschinellen Generierungsverfahrens gebildet wurde. Dabei sind lediglich die Ergebnisse zu Gebäuden und Wohnungen nicht mit einem

1 Eine detaillierte Beschreibung des Zensusmodells ist zu finden unter Statistische Ämter des Bundes und der Länder 2015. 
Zufallsfehler, sondern lediglich mit dem bei jeder statistischen Erhebung auftretenden systematischen Fehler behaftet. Bei den Personen trifft dies nur auf die demografischen Grunddaten spezieller Teilpopulationen zu, deren Daten im Rahmen einer Vollerhebung erfasst wurden.

Um die Daten aus den verschiedenen Erhebungsteilen verbinden zu können, wurde als erster Schritt ein Basisregister aufgebaut, das alle Anschriften mit Wohnraum enthielt, die es am Zensusstichtag (9. Mai 2011) in Deutschland gab. Dieses Anschriften- und Gebäuderegister (AGR) war das Bindeglied für alle Erhebungsteile während des gesamten Zensusablaufs und gleichzeitig die Grundgesamtheit für die Stichprobenerhebung bei privaten Haushalten und für die Gebäude- und Wohnungszählung. Den umfangsreichsten Adressdatenbestand für das AGR lieferten die Vermessungsbehörden, die sowohl Gebäude mit Wohnraum als auch rein gewerblich genutzte Gebäude verzeichnen. Hinzu kamen Adressdaten aus Melderegisterauszügen und aus den Registerdaten der Bundesagentur für Arbeit. Um sicherzustellen, dass alle am Stichtag zensusrelevanten Gebäude im AGR enthalten waren und damit einer systematischen Unterschätzung der Einwohnerzahl vorzubeugen, wurden alle Anschriften, die nur in einem der drei Datenbestände vorkamen, explizit daraufhin überprüft, ob an ihnen Wohnraum vorhanden war. Darüber hinaus wurde das AGR durch mehrere Datenlieferungen und durch Erkenntnisse aus den primärstatistischen Zensuserhebungen aktualisiert (Kleber et al. 2009).

Die Registerdaten der dezentral in den Gemeinden geführten Meldebehörden zu allen am Zensusstichtag gemeldeten Personen bildeten den Kern sowohl für die Ermittlung der Einwohnerzahl der Gemeinden als auch für die Daten über die demografische Struktur nach Geschlecht, Alter, Familienstand und Nationalität für die in privaten Haushalten lebende Bevölkerung. Sie wurden zum Zensusstichtag sowie nochmals drei Monate danach erhoben, um alle am Stichtag relevanten Eintragungen auch dann zu erfassen, wenn sie erst zeitlich verzögert im Register aufgenommen worden waren. Die Daten aus den Meldebehörden wurden zu einem gesamtdeutschen Datenbestand zusammengefasst und zunächst daraufhin überprüft, ob er Fälle enthielt, bei denen Personen am Stichtag mit mehr als einer alleinigen Wohnung oder Hauptwohnung gemeldet waren. Wurden solche Mehrfachfälle gefunden, wurden diese maschinell bereinigt, indem die jeweils aktuellsten zensusrelevanten Informationen übernommen wurden, sofern diese Mehrfachwohnsitze der betroffenen Personen nur in Gemeinden mit mindestens 10.000 Einwohnern (große Gemeinden) vorkamen. War jedoch eine mehrfach gemeldete Person in mindestens einer kleineren Gemeinde (mit weniger als 10.000 Einwohnern) gemeldet, fand die Bereinigung mit Hilfe der schriftlichen Befragung zur Klärung des Wohnsitzes statt, die auch immer dann eingesetzt wurde, wenn Personen nur mit Nebenwohnung gemeldet waren (Dieh/ 2012).

Für Bewohnerinnen und Bewohner an Anschriften mit Gemeinschaftsunterkünften, Anstalten, Wohnheimen und ähnlichen Einrichtungen wurden Angaben im Rahmen einer Vollerhebung ermittelt, da für sie die Melderegisterangaben aufgrund sehr hoher Fluktuation und unzureichendem Meldeverhalten zu viele Fehler enthalten. Dabei wurde unterschieden in Einrichtungen, bei denen die Kenntnis über diesen Wohnort für die betreffenden Personen die Gefahr einer sozialen Stigmati- 
Das Modell des Zensus 2011

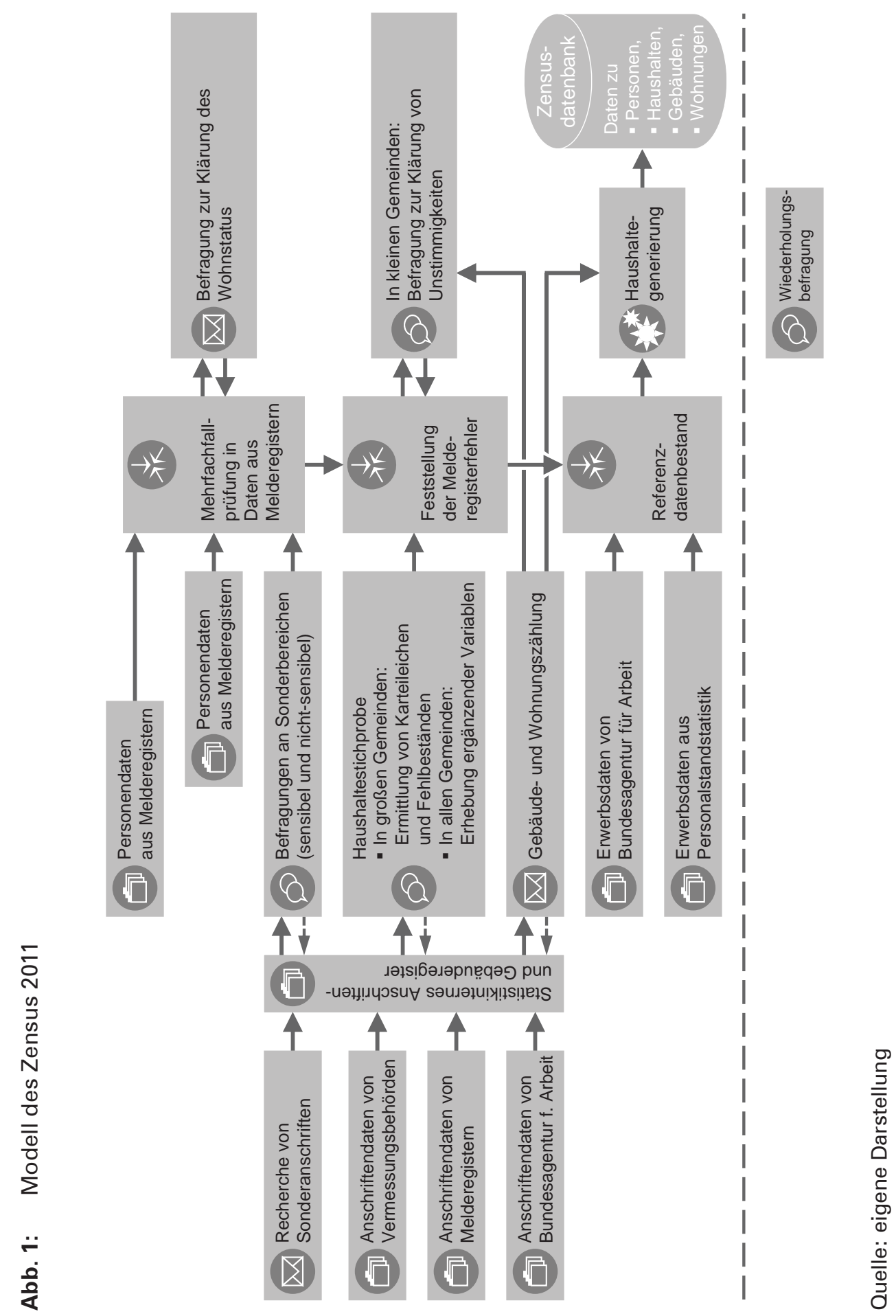


sierung enthielt - sogenannte sensible Sonderbereiche wie z.B. psychiatrische Kliniken - und Einrichtungen, für die das nicht der Fall war - sogenannte nicht-sensible Sonderbereiche wie z.B. Studierendenwohnheime. Auch die in den Sonderbereichserhebungen erfassten Personen wurden in die oben angesprochene Mehrfachfallüberprüfung einbezogen.

Eine Stichprobenerhebung in Privathaushalten bei knapp 10 \% der Bevölkerung sicherte die Qualität der um die Mehrfachfälle bereinigten Melderegisterdaten in Gemeinden mit mindestens 10.000 Einwohnern, indem gemeindespezifisch die Rate der Melderegisterfehler (Karteileichen- und Fehlbestandsraten) festgestellt und diese bei der Ermittlung der Bevölkerungszahl der Gemeinden berücksichtigt wurden. Außerdem wurden in dieser Stichprobenerhebung - in Gemeinden unter 10.000 Einwohnern und in den nicht-sensiblen Sonderbereichen - ergänzende Angaben über die Bevölkerung ermittelt, die nicht aus Registerangaben gewonnen werden konnten. ${ }^{2}$ Hierbei handelt es sich u.a. um Angaben zum Bildungsstand, zum Migrationshintergrund, zu Religion und Weltanschauung und zur Erwerbstätigkeit. Ausgewertet werden können diese ergänzenden Angaben aber nur bis zur Ebene der großen Gemeinden und für Kreise (NUTS-3-Regionen).

Um durch die Haushaltebefragung nicht nur Karteileichen, sondern auch Fehlbestände feststellen zu können, wurden in der Zufallsstichprobe jeweils ganze Anschriften ausgewählt und der Wohnstatus aller dort lebenden Personen mit den Melderegisterangaben zu dieser Anschrift verglichen. Auswahlgrundlage für diese Stichprobenziehung waren alle Anschriften aus dem AGR mit Ausnahme derjenigen, an denen es sensible Sonderbereiche gab.

Bei der Entwicklung des Stichprobenauswahlplans war anzustreben, dass die durch den Zensus festgestellten Einwohnerzahlen in großen Gemeinden mit $95 \%$ iger Sicherheit nur um $1 \%$ von der tatsächlichen Einwohnerzahl abweichen. Zur Optimierung der Stichprobenauswahl wurden die Anschriften der Grundgesamtheit mehrstufig geschichtet und ein Verfahren der Aufteilung des im Gesetz festgelegten Stichprobenumfangs auf die einzelnen Schichten eingesetzt, das die höchstmögliche Präzision für die Einwohnerzahlermittlung erwarten ließ. Hierbei konnte jedoch nur auf die vor dem Zensus vorliegenden Informationen über die Variabilität von Karteileichen und Fehlbeständen aus dem Zensustest von 2001 zurückgegriffen werden (Statistische Ämter des Bundes und der Länder 2004). Die tatsächliche Variabilität, die einen großen Einfluss auf die tatsächlich durch den Zensus 2011 erreichte Präzision hatte, wurde erst durch den Zensus selbst ermittelt. Das Optimierungsverfahren ergab für jede Gemeinde einen maßgeschneiderten Auswahlsatz, der zwischen 2,1\% und 45,6\% lag und sich auch bei vergleichbar großen Gemeinden deutlich unterscheiden konnte.

2 Das Stichprobendesign und das Hochrechnungsmodell für die Haushaltebefragung wurden in einem eigenen Forschungsprojekt durch die Universität Trier und GESIS - Leibniz-Institut für Sozialwissenschaften in Mannheim entwickelt (siehe hierzu Münnich et al. 2012). Die daraus für den Zensus 2011 abgeleiteten Verfahren sind beschrieben unter Berg/Bihler 2011 und Berg/ Bihler 2014a. 
Registerdaten der Bundesagentur für Arbeit zu sozialversicherungspflichtig Beschäftigten sowie Registerdaten zum Personalbestand der öffentlichen Arbeitgeber ergänzten auf Personenebene die demografischen Merkmale, die aus den Melderegistern resp. der Stichprobenbefragung in privaten Haushalten und in nichtsensiblen Sonderbereichen erhoben wurden, und bildeten mit diesen und dem Anschriften- und Gebäuderegister den Referenzdatenbestand (RDB) (Hirner/Stig/mayr 2013). Durch die Bildung des RDB konnten die Angaben aus allen Datenquellen erhebungsteilübergreifend geprüft werden und erhielten ihren räumlichen Bezug, der nicht nur administrative Einheiten umfasst, sondern auch eine deutschlandweite Gittereinteilung mit einer Rastergröße von 100 mal 100 Metern.

Die personenscharfe Zusammenführung der Datensätze aus den verschiedenen Datenquellen war eine der großen Herausforderungen beim Zensus 2011, da sie ohne bereits vorhandene Identifikationsnummern durchgeführt werden musste. Die Identifikation von zusammengehörenden Datensätzen erfolgte maschinell über personen- und anschriftenbezogene Merkmale, also mit Hilfe von Namen, Geschlecht, Geburtsdatum, amtlichem Gemeindeschlüssel, Postleitzahl, Straße und Hausnummer. Nur etwa $1 \%$ der Datensätze konnten maschinell nicht verknüpft, sondern mussten manuell nachbearbeitet werden.

Angaben zur Anzahl und Struktur von Wohngebäuden und Wohnungen wurden durch eine postalische Befragung bei allen Eigentümern erhoben. Zu den Gebäuden wurden Fragen nach der Art des Gebäudes, dem Baujahr, der Zahl der Wohnungen und der Heizungsart gestellt, bei Wohnungen wurde unter anderem nach der Größe, der Zahl der Räume und der Wohnungsnutzung gefragt (Statistische Ämter des Bundes und der Länder 2014). Gab es in Gemeinden mit weniger als 10.000 Einwohnern aus dem Vergleich der Angaben aus der Gebäude- und Wohnungszählung und der Angaben aus den Melderegistern Hinweise auf Unter- oder Übererfassungen, wurde diesen mit der schriftlichen Befragung zur Klärung von Unstimmigkeiten nachgegangen. Dieses Erhebungsinstrument kam jedoch nur bei Einfamilienhäusern zum Einsatz, da nur dort ein günstiges Verhältnis von Bereinigungseffekt und Befragungsaufwand zu erwarten war und da diese Maßnahme eine vergleichbare Qualitätssicherung der aus den Melderegistern stammenden Daten versprach, wie sie bei Gemeinden ab 10.000 Einwohnern durch die Stichprobe erreicht wurde.

Um auch Ergebnisse darüber zu erhalten, wie Personen in Haushalten zusammenleben und wie deren Wohnsituation aussieht, wurden mit einem maschinellen Verfahren der Haushaltegenerierung Personendaten zu statistisch generierten Haushalten von zusammenwohnenden Personen verknüpft. Hierzu wurden für jede Anschrift Meldedaten, die auch Informationen über Familienzusammenhänge enthielten, mit Angaben aus der Gebäude- und Wohnungszählung automatisiert zusammengeführt.

Zur Bewertung der für Gemeinden ab 10.000 Einwohnern ermittelten Einwohnerzahlen gab es schließlich noch eine Wiederholungsbefragung, die durch Erhebungsbeauftragte bei etwa $5 \%$ der Anschriften aus der Haushaltebefragung durchgeführt wurde (Klink/Bihler 2015). 


\section{Besonderheiten der Zensusdaten}

Die Daten des Zensus wurden mit Auskunftspflicht erhoben, lediglich eine Frage zur Weltanschauung war nicht verpflichtend zu beantworten. Trotzdem mussten die Daten überprüft und ggf. korrigiert oder ergänzt werden, wenn sie als unplausibel erkannt wurden oder ganz fehlten. Hierzu wurden sowohl Cold-Deck- und Hot-Deckals auch deterministische Imputationsverfahren eingesetzt. Bei der Gebäude- und Wohnungszählung lagen nach dem Dateneingang 31 \% aller Fragebogen vollständig plausibel vor. Das Ausmaß der notwendigen Imputationen war insbesondere für das Merkmal Eigentumsverhältnis hoch, das für 18,5 \% der Gebäude und für 45,3\% der Wohnungen ersetzt werden musste. ${ }^{3}$ Bei den übrigen Wohnungsfragen lagen die Imputationsraten unter 16 \% (Grundwald/Krause 2014). Bei der Haushaltebefragung war die Hälfte aller Fragebogen plausibel beantwortet. Bezogen auf die einzelnen Themen hatten die Fragen zur Erwerbstätigkeit mit $25 \%$ unplausibler Datensätze die höchsten Imputationsraten (Statistisches Bundesamt 2016: 31). Die freiwillige Frage zur Weltanschauung wurde nur von $48 \%$ derjenigen Personen beantwortet, die angaben, keiner öffentlich-rechtlichen Religionsgemeinschaft anzugehören. Da angesichts dieser hohen Item-Nonresponse-Rate von einer großen Verzerrung ausgegangen werden muss, hat das Statistische Bundesamt darauf verzichtet, diese Frage auszuwerten.

Die mit dem Zensus 2011 ermittelten Einwohnerzahlen der großen Gemeinden ergeben sich aus zwei Teilen. Die an Anschriften mit Sonderbereichen durch eine Vollerhebung ermittelten Einwohner wurden ausgezählt (Auszählungsteil). Für alle übrigen Anschriften wurde der um Mehrfachfälle bereinigte Melderegisterbestand um die aus der Stichprobe hochgerechneten Über- und Untererfassungen korrigiert (Korrekturteil) (Berg/Bihler 2014b). Für diese Anschriften wurde zunächst die Zahl der zur Stichtagsbevölkerung gehörenden Personen und die Zahl der Personen, die im Melderegister korrekt verzeichnet waren, geschätzt und mit diesen beiden Werten die Karteileichen und Fehlbestände der jeweiligen Gemeinden abgeleitet. Stichprobenmethodisch war dieses Vorgehen günstiger als die direkte Schätzung der Unter- und Übererfassung, da es sich hierbei um seltene Ereignisse handelt und hierfür der Schätzfehler größer wäre. Die Schätzung des Korrekturteils der Einwohnerzahl erfolgte mit einem verallgemeinerten Regressionsschätzer jeweils simultan für alle Auswahlschichten der einzelnen Gemeinden. Summiert mit dem Auszählungsteil ergibt sich so die Einwohnerzahl der großen Gemeinden. Teil des Hochrechnungsverfahrens war auch die Varianzschätzung um zu überprüfen, inwieweit das angestrebte Präzisionsziel für die Einwohnerzahlermittlung erreicht werden konnte. Tatsächlich konnte das Ziel nur in $37 \%$ der Gemeinden erreicht werden, der mittlere Wert lag bei 0,56 \% einfachem relativem Standardfehler. Ursache hierfür

3 Bei der Frage nach dem Eigentumsverhältnis der Wohnung wurde häufig nicht verstanden dass diese Frage nur für Eigentumswohnungen auszufüllen war, d.h. der hohe Wert ist durch die Filterführung im Fragebogen zu erklären, die auf die Qualität der übrigen Erhebungsmerkmale keinen Einfluss hat. 
war, dass die Annahmen, die der Entwicklung des Stichprobendesigns zugrunde lagen, zu positiv waren (Statistisches Bundesamt 2016: 7).

Für die kleinen Gemeinden ergeben sich die aus dem Zensus 2011 ermittelten Einwohnerzahlen ebenfalls aus der Auszählung der an Anschriften mit Sonderbereichen wohnenden Personen, die auch in diesen Gemeinden vollständig erhoben wurden, sowie aus der Auszählung des bereinigten Melderegisterbestands, der um die Über- und Untererfassungen korrigiert wurde, die mit der Befragung zur Klärung von Unstimmigkeiten festgestellt worden waren.

Um die personenbezogenen Zensusdaten richtig interpretieren zu können, müssen jeweils die Datenquellen berücksichtigt werden, aus denen sie stammen. Die Merkmale, die nicht aus Registern, sondern nur aus der Haushaltebefragung gewonnen werden konnten, liegen nur für Personen in Privathaushalten und für Personen in nicht-sensiblen Sonderbereichen vor. Werden diese Stichprobenmerkmale zusammen mit demografischen Grundmerkmalen ausgewertet, stammen letztere auch aus den Stichprobendaten und können daher in den Randsummen von denjenigen Ergebnissen abweichen, die aus den Registermerkmalen ausgewertet werden. Die erwerbsstatistischen Ergebnisse stammen zum Teil aus Registern und zum Teil aus der Haushaltestichprobe und der Erhebung an Anschriften mit nicht-sensiblen Sonderbereichen. Die Merkmale Erwerbsstatus, Stellung im Beruf und Wirtschaftszweig werden für sozialversicherungspflichtig Beschäftigte und alle Beschäftigten des öffentlichen Dienstes ausgezählt und für alle übrigen Erwerbstätigen aus der Stichprobe hochgerechnet. Werden die genannten Merkmale mit anderen Merkmalen aus der Stichprobe gemeinsam ausgewertet, werden alle Ergebnisse aus den Stichprobendaten geschätzt, was zu Unterschieden zu den rein registerbasierten Ergebnissen führen kann (Sedmihradsky et al. 2012).

Die Daten, die aus Vollerhebungen oder aus Registern stammen, können zur Ergebniserstellung ausgezählt werden, während die Daten aus der Haushaltestichprobe hochgerechnet werden müssen. Damit ergeben sich auch unterschiedliche Anforderungen an diejenigen Verfahren, die zur statistischen Geheimhaltung dieser Daten angewandt werden. Die Ergebnisse der Gebäude- und Wohnungszählung und der Haushaltegenerierung sowie die Daten aus den Melderegistern und aus der Erhebung an Anschriften mit nicht-sensiblen Sonderbereichen werden durch das datenverändernde Verfahren SAFE geheim gehalten (Gießing et al. 2014). Dabei wird für jede Gemeinde und für Berlin und Hamburg für die jeweiligen Stadtteile sichergestellt, dass jede personenbezogene Merkmalskombination und jede Merkmalskombination aus der Gebäude- und Wohnungszählung und der Haushaltegenerierung jeweils mindestens dreimal auftritt. Abweichend von diesem Vorgehen werden die Gesamteinwohnerzahlen der Gemeinden ohne Datenveränderung ermittelt. Auch Verhältniszahlen, die von den statistischen Ämtern veröffentlicht werden, werden aus unveränderten Daten berechnet. Die statistische Geheimhaltung erfolgt erst anschließend durch Vergröberung des Divisionsergebnisses.

Die hochgerechneten Ergebnisse werden keinem gesonderten Geheimhaltungsverfahren unterzogen. Haben einzelne Ergebnisse einen relativen Standardfehler von mehr als $15 \%$ und sind damit unzuverlässig, werden sie nicht veröffentlicht. Setzen sich Ergebnisse aus einem ausgezählten und einem hochgerechneten Teil 
zusammen, wird bei der Sperrung eines Ergebnisses berücksichtigt, welchen Anteil des Ergebnisses die ausgezählten und die hochgerechneten Ergebnisteile haben. Alle Ergebnisse, die hochgerechnet wurden oder einen Hochrechnungsanteil haben, werden auf ein Vielfaches von 10 gerundet, um deutlich zu machen, dass es sich um keine exakten Werte handelt. Durch die Kombination von Stichprobenzufallsfehler, Sperrung von unzuverlässigen Ergebnissen und Rundung ist die statistische Geheimhaltung gewährleistet.

Die demografischen Ergebnisse des Zensus 2011 bilden in ihrer Untergliederung nach Geschlecht, Altersjahren, Familienstand und Staatsangehörigkeit für jede Gemeinde die Grundlage für die Ermittlung des Bevölkerungsstandes bis zum nächsten Zensus. Für die Berechnung der fortschreibungsrelevanten Untergliederungen der Einwohnerzahlen wurde ein spezifisches Verfahren verwendet, das zu Abweichungen in den demografischen Strukturen gegenüber dem Zensusergebnis für die einzelnen Gemeinden führen konnte. Die Einwohnerzahl insgesamt der einzelnen Gemeinden wurde dadurch jedoch nicht verändert. Notwendig wurde dieses Vorgehen, da das Hochrechnungsverfahren des Zensus 2011 auf eine möglichst präzise Ermittlung der Gesamteinwohnerzahl der Gemeinden ausgerichtet war. Für die tiefe demografische Untergliederung führte dieses Verfahren jedoch in einigen Gemeinden zu auffälligen Ergebnissen in einigen Kombinationen von Altersstufe und Geschlecht. Wären diese Auffälligkeiten nicht bereinigt worden, hätte dies nicht nur die laufende Bevölkerungsfortschreibung bis zum nächsten Zensus belastet, sondern auch die Berechnung demografischer Maße wie Geburten- und Sterbeziffern (Statistisches Bundesamt 2015).

\section{Literatur}

Berg, Andreas; Bihler, Wolf 2011: Das Stichprobendesign der Haushaltsstichprobe des Zensus 2011. In: Wirtschaft und Statistik 04/2011: 317-328 [https://www.destatis.de/DE/Publikationen/WirtschaftStatistik/Monatsausgaben/WistaApril11.pdf? blob= publicationFile, 25.07.2016].

Berg, Andreas; Bihler, Wolf 2014a: Der Auswahlplan für die Ziehung der Neuzugänge der Haushaltsstichprobe des Zensus 2011. In: Wirtschaft und Statistik 03/2014: 151154 [https://www.destatis.de/DE/Publikationen/WirtschaftStatistik/Monatsausgaben/ WistaMaerz2014.pdf?_blob=publicationFile, 25.07.2016].

Berg, Andreas; Bihler, Wolf 2014b: Das Hochrechnungsverfahren zur Ermittlung der Einwohnerzahl im Zensus 2011. In: Wirtschaft und Statistik 04/2014: 229-235 [https:// www.destatis.de/DE/Publikationen/WirtschaftStatistik/Monatsausgaben/WistaApril2014.pdf? blob=publicationFile, 25.07.2016].

Diehl, Eva-Maria 2012: Methoden der Mehrfachfallprüfung im Zensus 2011. In: Wirtschaft und Statistik 06/2012: 473-484 [https://www.zensus2011.de/SharedDocs/Downloads/ DE/Publikationen/Aufsaetze_Archiv/2012_06_Destatis_Methoden_der_Mehrfachfallpruefung_im_Zensus_2011.pdf?_blob=püblicationFilęgv=14, 25.07.2016].

Gießing, Sarah et al. 2014: Geheimhaltung beim Zensus 2011. In: Wirtschaft und Statistik 11/2014: 641-647 [https://www.destatis.de/DE/Publikationen/WirtschaftStatistik/ Monatsausgaben/WistaNovember2014.pdf? blob=publicationFile, 25.07.2016]. 
Grundwald, Sven; Krause, Anja 2014: Umgang mit fehlenden Angaben in der Gebäudeund Wohnungszählung 2011. In: Wirtschaft und Statistik 08/2014: 437-449 [https:// www.destatis.de/DE/Publikationen/WirtschaftStatistik/Monatsausgaben/WistaAugust2014.pdf?__blob=publicationFile, 25.07.2016].

Hirner, Stephanie; Stiglmayr, Susanne 2013: Der Referenzdatenbestand im Zensus 2011. In: Wirtschaft und Statistik 01/2013: 30-41 [https://www.destatis.de/DE/Publikationen/ WirtschaftStatistik/Monatsausgaben/WistaJanuar2013.pdf? blob=publicationFile, 25.07.2016].

Kleber, Birgit et al. 2009: Aufbau des Anschriften- und Gebäuderegisters für den Zensus 2011. In: Wirtschaft und Statistik 07/2009: 629-640 [https://www.destatis.de/DE/Publikationen/WirtschaftStatistik/Monatsausgaben/WistaJuli09.pdf? blob= publicationFile, 25.07.2016].

Klink, Steffen; Bihler, Wolf 2015: Die Wiederholungsbefragung beim Zensus 2011. In: Wirtschaft und Statistik 02/2015: 42-54 [https://www.destatis.de/DE/Publikationen/ WirtschaftStatistik/2015/02/Wista_2_2015.pdf?_blob=publicationFile, 25.07.2016].

Münnich, Ralf; Gabler, Siegfried et al. 2012: Stichprobenoptimierung und Schätzung in Zensus 2011. Statistik und Wissenschaft Band 21. Wiesbaden: Statistisches Bundesamt [https://www.destatis.de/DE/Publikationen/StatistikWissenschaft/Band21 StichprobenoptimierungZensus1030821129004.pdf? blob=publicationFile, 25.07.2016].

Sedmihradsky, Dirk; Eisenmenger, Matthias; Burmeister, Friederike 2012: Erhebung, Aufbereitung und Zusammenführung der Erwerbsregisterdaten im Zensus 2011. In: Wirtschaft und Statistik 12/2012: 1052-1064 [https://www.destatis.de/DE/Publikationen/ WirtschaftStatistik/Monatsausgaben/WistaDezember12.pdf?_blob=publicationFile, 25.07.2016].

Statistische Ämter des Bundes und der Länder 2004: Ergebnisse des Zensustests. In: Wirtschaft und Statistik 08/2004: 813-833 [https://www.destatis.de/DE/Publikationen/ WirtschaftStatistik/Monatsausgaben/WistaAugust04.pdf?__blob=publicationFile, 25.07.2016].

Statistische Ämter des Bundes und der Länder 2014: Zensus 2011 - Gebäude und Wohnungen. Übersicht über Merkmale und Merkmalsausprägungen, Definitionen [https:// www.zensus2011.de/SharedDocs/Downloads/DE/Merkmale/Merkmale_GWZ.pdf? blob= publicationFile\&v=13, 25.07.2016]

Statistische Ämter des Bundes und der Länder 2015: Zensus 2011 - Methoden und Verfahren. Wiesbaden 2015 [https://www.zensus2011.de/SharedDocs/Downloads/ DE/Publikationen/Aufsaetze Archiv/2015 06_MethodenUndVerfahren.pdf? blob $=$ publicationFile\&v $=6,25.07 .2016]$.

Statistisches Bundesamt 2015: Ausgangsdaten der Bevölkerungsfortschreibung aus dem Zensus 2011 [https://www.destatis.de/DE/Publikationen/Thematisch/Bevoelkerung/Bevoelkerungsstand/DatenBevoelkerungsfortschreibungZensus.html, 25.07.2016].

Statistisches Bundesamt 2016: Qualitätsbericht nach § 17 ZensG2011. Wiesbaden: Statistisches Bundesamt [https://www.zensus2011.de/SharedDocs/Downloads/ DE/Publikationen/Aufsaetze_Archiv/2016_05_Zensus2011_Qualitaetsbericht.pdf? blob= publicationFile\& $v=8,2 \overline{5} .07 .2016]$.

Dr. Sabine Bechtold $(\bowtie)$. Statistisches Bundesamt. Wiesbaden, Deutschland.

E-mail: sabine.bechtold@destatis.de

URL: https://www.destatis.de/DE/Startseite.html 


\section{Comparative Population Studies}

wWW.comparativepopulationstudies.de

ISSN: 1869-8980 (Print) - 1869-8999 (Internet)

\section{Published by}

Prof. Dr. Norbert F. Schneider

Federal Institute for Population Research D-65180 Wiesbaden / Germany

\section{(cc) BY-SA}

2017

\section{Managing Editor}

Frank Swiaczny

\section{Assistant Managing Editor}

Katrin Schiefer

\section{Copy Editor}

(Selected Articles in German)

Dr. Evelyn Grünheid

\section{Layout}

Beatriz Feiler-Fuchs

E-mail: cpos@bib.bund.de

\section{Scientific Advisory Board}

Paul Gans (Mannheim)

Karsten Hank (Cologne)

Johannes Huinink (Bremen)

Michaela Kreyenfeld (Rostock)

Marc Luy (Vienna)

Notburga Ott (Bochum)

Peter Preisendörfer (Mainz)

Nikola Sander (Groningen)

Zsolt Spéder (Budapest)

\section{Board of Reviewers}

Martin Abraham (Erlangen)

Laura Bernardi (Lausanne)

Hansjörg Bucher (Bonn)

Claudia Diehl (Konstanz)

Andreas Diekmann (Zurich)

Gabriele Doblhammer-Reiter (Rostock)

Jürgen Dorbritz (Wiesbaden)

Anette Eva Fasang (Berlin)

E.-Jürgen Flöthmann (Bielefeld)

Alexia Fürnkranz-Prskawetz (Vienna)

Beat Fux (Salzburg)

Joshua Goldstein (Berkeley)

Sonja Haug (Regensburg)

Hill Kulu (Liverpool)

Aart C. Liefbroer (The Hague)

Kurt Lüscher (Konstanz)

Emma Lundholm (Umeå)

Nadja Milewski (Rostock)

Dimiter Philipov (Vienna)

Roland Rau (Rostock)

Tomáš Sobotka (Vienna)

Jeroen Spijker (Barcelona)

Olivier Thévenon (Paris)

Helga de Valk (Brussels)

Heike Trappe (Rostock)

Michael Wagner (Cologne) 